\title{
Research \& Development of E-Map Service System based on Baidu Map API for Regional Seismic Network
}

\author{
Liuli $^{1, a}$, DongYibing ${ }^{1,2, b}$ and LiuXin ${ }^{1,2, c}$ \\ ${ }^{1}$ Seismological Bureau of Hebei Province, Shijiazhuang,Hebei,China \\ ${ }^{2}$ School of Earth and Space Sciences of USTC, Hefei, Anhui,China \\ aliuli@eq-he.ac.cn, byuehun06@yahoo.cn , chbdsn_Ix@163.com
}

Keywords: Regional Seismic Network, E-Map, Baidu Map API

\begin{abstract}
In this paper, new $\mathrm{B} / \mathrm{S}$ management information software developed for the regional seismic network is introduced. The main function of the software is to provide e-map service for two types of clients: administrators and guests. The application of the software will change the traditional ways and improve the working efficiency of the maintenance engineers. It was developed with Eclipse and MySQL database, following MVC framework specification. It should be deployed in the center of the regional seismic network, providing e-map services over Internet for clients. It can also provide certain dedicated functions for users according to their privileges. It has been proved that the software has both useful functions and reliable performance. The e-map service is not only helpful for the maintenance of regional seismic network, but also beneficial to the emergency relief after strong earthquake.
\end{abstract}

\section{Introduction}

In recent years, with the development of our country's cause of earthquake monitoring, the size of the regional seismic network is constantly expanding, and the density of the seismic stations is increasing, leading to an increasing workload of the network maintenance. At present, the seismic station information management is by means of MS Office software and some paper documents without any dedicated management information system. It is such a heavy load for the maintenance engineers to clearly remember the location and driving directions of each station. When they are going to a station where they have not been ever before, they usually find the help of free e-map and the GPS. However, these services are useless if they have forgotten the location especially when they are in the wild. With the further development of the seismic network, the traditional ways will be difficult to adapt to the needs. In order to meet this demand, we have to design a dedicated online e-map service, which will support functions in the $\mathrm{B} / \mathrm{S}$ mode both for administrators and authorized guests.

This system will run at the center of the regional seismic network as a web server. Remote user can access to the system through the computer's Web browser, searching the station he is going to and querying the best route from his position to the destination. As a result, it will greatly reduce the workload of the maintenance engineers, so that they can pay more attention to their owns work, thereby enhancing their working efficiency and quality. Besides, the system will also provide e-map service for the authorized guests. So it will also be beneficial to the emergency relief after strong earthquake.

\section{Requirements Analysis}

Functional Requirements. For the administrators, the system should support three main functions: seismic network information management, seismic network e-map service and system user management. Seismic network information management should support operations to the station record in the database like adding, deleting, modifying, querying, printing and so on. Seismic network e-map service should support the common operations of Baidu Map such as panning, zooming, and route planning and some dedicated functions like seismic station marks and info windows. System 
user management should support operations to the user record in the database like adding, deleting, modifying, querying and so on. For the guests, the system should support two main functions: seismic network e-map service and personal account management.

Performance Requirements. The performance of the system should meet the following basic requirements: Good user interface to reduce the operators' workload; Good operating efficiency to improve the staffs' work; Good scalability to adapt to the extensions of the seismic network in the future; Good flexibility to adapt to the changes in the configuration; Good security to prevent unauthorized access to internal content; Good maintainability and cross-platform features.

Running Environments. The system will run at B/S mode, so it contains two sides communicating over the network: server and client. Each side needs certain network, hardware and software environment.

Network Environment. Server should be deployed in the center of the regional seismic network, providing the online e-map service by way of calling Baidu Map API from the Baidu Map servers. Clients could access to the server with their web browser. The topological structure of the system is shown in Fig. 1.

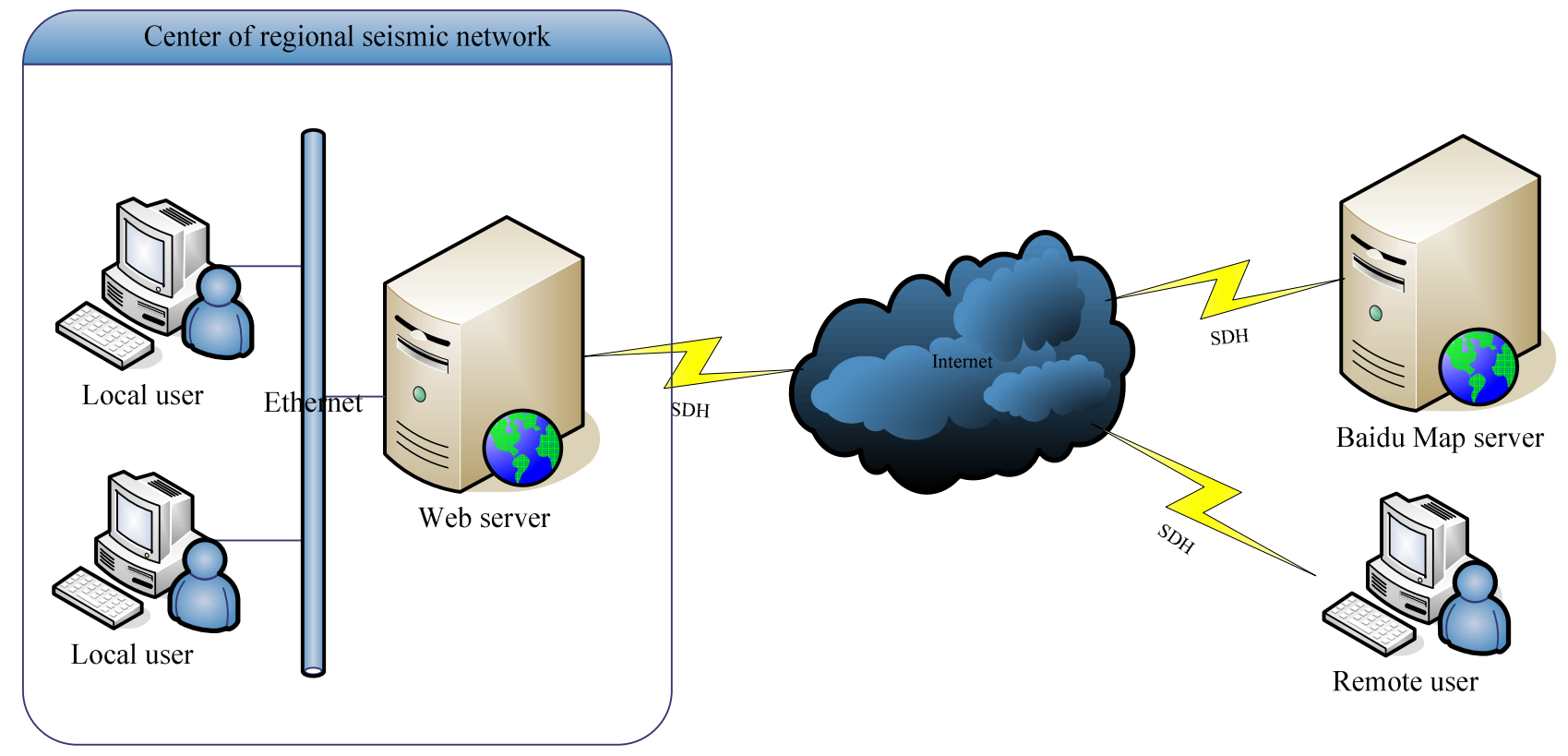

Fig. 1 Topological Structure of the System

Hardware Environment. As to the server, there are some requirements as following: CPU: P4 2.0GHz or above; Memory: $1 \mathrm{G}$ or above; Network Card: Gigabit Ethernet; Hard disk: 80G. As to the client, an ordinary PC can play well.

Software Environment. The server should build the following software environment: OS: Windows XP or above / UNIX / Linux; JVM: jre 1.6; DBMS: MySQL 5.1; Web server: Tomcat 6.0.35; Web browser: IE 6.0 or above. And the client need these software: OS: Windows XP or above / UNIX / Linux; JVM: jre 1.6; Web browser: IE 6.0 or above.

\section{Software Design}

Interface Design. The software of server can be divided into two subsystems according to the different privilege of the users: administrator subsystem and guest subsystem. Administrator subsystem is designed for the administrators of the seismic network. Guest subsystem is designed for the authorized guests out of the seismic network. For the administrators, we have designed these pages: Login page: checking the users' name and password, providing the registration entrance for new users; Main page: providing hyperlinks to administrational functions including seismic network basic information management, seismic network e-map application and system user management; Seismic network basic information management page: providing administrational functions including 
seismic station displaying, adding, deleting, modifying, querying, sorting, saving as and so on; Seismic network e-map service: supporting the common operations of e-map such as panning, zooming, and route planning and some dedicated functions like seismic station marks and info windows; System user management: providing administrational functions including system user displaying, adding, deleting, modifying, querying and so on. For the guests, we have designed these pages: Login page: same as above; Main page: providing hyperlinks to functions including seismic network e-map application and personal account management; Seismic network e-map service: same as above; Personal account management: supporting functions like changing password and logging out.

Database Design. We have chosen MySQL as the DBMS and Navicat for MySQL as the tool. We have designed two tables in the database: one is the table of seismic network basic information, which includes records of all the station belonging to the network, whose structure is shown in Table 1 . The other is the table of system user information, which includes records of all the system users including the administrators and guests, whose structure is shown in Table 2.

Table 1 Table of Seismic Network Basic Information

\begin{tabular}{ccc}
\hline Field Name & Data Type & Description \\
\hline StationID & Int(10) & Identification of the station; Key field \\
StationName & VarChar(10) & Name of the station \\
StationCode & VarChar(10) & Code of the station \\
Measurement & VarChar(10) & Measurement type of the station \\
Platform & VarChar(20) & Platform type of the station \\
Site & VarChar(20) & Site type of the station \\
Longitude & VarChar(10) & Longitude of the station \\
Latitude & VarChar(10) & Latitude of the station \\
Height & VarChar(10) & Height of the station \\
Seismographer & VarChar(20) & Seismographer type of the station \\
Digitizer & VarChar(20) & Digitizer type of the station \\
Link & VarChar(10) & Link type of the station \\
Power & VarChar(20) & Power type of the station \\
Location & VarChar(20) & Location of the station \\
\hline
\end{tabular}

Table 2 Table of System Users

\begin{tabular}{ccc}
\hline Field Name & Data Type & Description \\
\hline UID & Int(10) & Identification of the user; Key field \\
Name & VarChar(20) & Name of the user \\
Password & VarChar(20) & Password of the user \\
Privilege & VarChar(20) & Privilege of the user: administrator or guest \\
\hline
\end{tabular}

\section{Software Implement}

Development Environment. The software is developed under the following environment and tools: OS: Windows XP SP3; JDK: SDK 1.6.0; IDE: Eclipse 3.3.5 + MyEclipse 6.0.1; Web Server: Tomcat 6.0.35; DBMS: MySQL 5.1.

\section{Development Technologies.}

Development Framework. To improve the maintainability, we have used MVC framework to design the overall structure of the software. MVC is the abbreviation of three words, namely: Model, View and Controller. The purpose of MVC is to divide the whole web system into low-coupled modules according to different functions [1]. Model is the implementation of the business logic, usually developed with JavaBean or EJB. View is the interaction with the users, usually with JSP. Controller is a bridge of communication between Model and View, it can dispatch the user's request and select the appropriate view for display, and it also can explain the user's inputs and Map them to operations that Model can perform. For our software, we have chosen JSP for View and JavaBean for Model.

E-map Development. For our e-map service, we have chosen Baidu Map API V1.3. Baidu Map API is provided in JavaScript; it includes not only the basic interface to build a map, but also provides data services such as local searching and route planning [2]. Nowadays, there are so many e-map services widely used such as Baidu Map, Google Map, and Bing Map etc. Most of the vendors 
provide both online and offline e-map service. After investigation, we have chosen Baidu Map API which is stated as open resource by Baidu Map to build our e-map service. Our choice is based on the following considerations: First is free of charge. Baidu Map API is free of charge for the public service sites. Developers can use functions such as picture displaying, location searching, route planning and real-time traffic checking, as long as they are willing to comply with the restraints(the direct access or use of internal data is not allowed, and the copyright information must be retained, etc.). Second is regularly updated. Baidu Map have been maintaining the half-yearly bulk data updates and the occasional local data updates, which eliminates the trouble of regularly manually downloading and installing the offline map pack. Third is supporting plug-in development. Baidu Map provides detailed development guidelines and samples on its website. All that the users need to do is just call the API to achieve their own purposes. Forth is authorized. Baidu Map has been authorized to operate e-map services by the national administration of surveying, mapping and geoinformation. These features make it very suitable to be used to build a small, dedicated WebGIS platform [3].

Development Roadmap. The development roadmap mainly includes the following steps: (1) To build the overall framework of the software. Where, JSP is used to design front pages, JavaBean is used to implement business logics, and DAO is used to deal with the connection between pages and database. In this way, to complete the development of other modules except the e-map service. (2) To build the map. Baidu Map API scripts can be embedded in the JSP page to build the map. In the same way, we can add some controls to the map to enhance its functionality. (3) To add seismic network dedicated functions to the map. Extracting the latitude and longitude of seismic stations from the database and adding them to the map. As a result, the station on the map will keep synchronized with the database. To add other dedicated applications such as station info window and complete the development of e-map service. (4) To modify the pages to make the interface more friendly.

\section{Conclusions}

In this paper, the research and development of an e-map service system for the regional seismic network is introduced. The application of the Java EE technology greatly enhanced the maintainability of the system, and the utility of Baidu Map API lead to an agile development. It has been proved that the utility of the system will effectively reduce the workload of the maintenance engineers and improve their work efficiency. Besides, the e-map service is not only helpful for the maintenance of regional seismic network, but also beneficial to the emergency relief after strong earthquake. Therefore, the system has a good prospect.

Due to limitations of manpower, material and financial resources, there are still some shortcomings in the current system, and there is still much room for improvement. In the future, to meet the new needs of the growing seismic network, we will continue to fix the defects, enrich the functions, and optimize the performance of the system.

\section{References}

[1] James L, Weaver, Kevin Mukhar, et a1, Beginning J2EE 1, From Movice to Professional Press, 2007, pp.280-310.

[2] Information on http://dev.baidu.com/wiki/map/.

[3] Du Mingchuan, Application of Baidu Map API in Small Geographic System, Geomatics \& Spatial Information Technology, 2011, 34(2) 152-156. 